Christoph Bachmann

\section{Einleitung}

Die Natur geht mit unseren Verdauungsbeschwerden "gnädig» um, stellt sie doch eine Reihe von Arzneipflanzen zur Verfügung, die bei verschiedenen Arten von Beschwerden des Gastrointestinaltrakts wirksam sind. Diese Arzneipflanzen werden als Tees, Tinkturen oder Trockenextrakte in Form von Tabletten eingenommen. Dazu gehören Kamille (Matricaria recutia), Pfefferminze (Mentha piperita), Enzian (Gentiana lutea), Tausendgüldenkraut (Centaurium umbellatum), Kalmus (Acorus calamus), Schafgarbe (Achillea millefolium), Löwenzahn (Taraxacum officinale), Mariendistel (Silybum marianum) und viele weitere mehr. Die Wirksamkeit dieser Arzneipflanzen gegen Verdauungsprobleme wurde anhand zahlreicher Studien dokumentiert.

\section{Artischocke}

Bei diesen Diskussionen stehen Extrakte aus Artischockenblättern eher nicht im Vordergrund. Die Artischocke (Cynara scolymus oder Cynara cardunculus) (Abb. 1) ist mehr als Gemüse bekannt, stellt jedoch auch eine sehr wertvolle Arzneipflanze dar, die

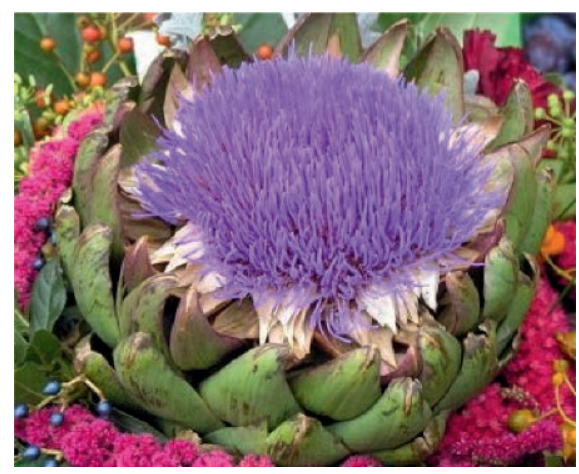

Abb. 1. Artischocke mit geöffnetem Blütenstand.

\title{
Studien bestätigen die Wirksamkeit von Extrakten aus Artischockenblättern bei funktionellen Verdauungsbeschwerden
}

bei funktionellen Dyspepsien und manchmal auch zur Senkung der Lipidwerte wertvolle Dienste leisten kann.

\section{Botanik}

Die Artischocke gehört zu den Korbblütlern (Asteraceae) und ist eine distelartige, ausdauernde Pflanze, die im ersten Vegetationsjahr eine grundständige Rosette bildet. Dann erscheint sie etwa 5 Jahre lang in Form von bis zu $2 \mathrm{~m}$ hohen Stängeln mit den bekannten Blütenständen, deren Böden fleischig sind und als Gemüse gegessen werden. Ihre ursprünglichen Verbreitungsgebiete sind die Kanarischen Inseln, der Mittelmeerraum, Nordafrika und Gebiete östlich des Mittelmeers bis Persien. Die heute kultivierten Artischockensorten weisen im Gegensatz zu den wild wachsenden kaum Dornen auf.

\section{Inhaltsstoffe}

Für die pharmakologische Wirksamkeit der Artischocke werden heute drei Stoffgruppen verantwortlich gemacht, die vor allem in den Blättern in unterschiedlichen Konzentrationen vorkommen [1]:

- Flavonoide (z.B. Luteolin-Glykoside wie Cynarosid),

- Hydroxyzimtsäuren,

- Sesquiterpenlactone wie Cynaropikrin (Abb. 2).

\section{Pharmakologie}

Pharmakologische Untersuchungen an Tieren und an freiwilligen Probanden zeigten, dass Artischockenextrakt eine erhebliche Steigerung der Cholerese bewirkt [2, 3]. Weiter

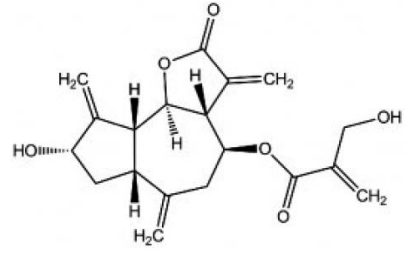

Abb. 2. Cynaropikrin, das der Artischocke den bitteren Geschmack gibt.

konnte auch in Tierversuchen eine Verstärkung der Cholesterinausscheidung [4] und in vitro zusätzlich eine Hemmung der Biosynthese von Cholesterin beobachtet werden [5].

\section{Steigerung der Cholerese}

Die Untersuchungen von Kirchhof et al. [2] wurden als placebokontrollierte Doppelblindstudie angelegt und mit 20 freiwilligen Probanden durchgeführt. Die Verum- (Gruppe A) und die Placebogruppe (Gruppe B) bestand je aus 10 Probanden, d.h. Männer mit einem mittleren Alter von 24,4 (A) bzw. 27,5 Jahren (B) und einem mittleren Körpergewicht von 74,6 (A) bzw. 77,5 kg (B), die alle weder regelmässige Oberbauchbeschwerden aufwiesen noch starke Raucher oder Kaffeetrinker waren und auch keine Stuhlanomalien aufwiesen.

Nach einer achttägigen Vorphase wurde an einem Tag (Prüfphase I) die Basissekretion gemessen und anschliessend mittels intraduodenaler Applikation während 1 h 6 Kapseln des Prüfpräparats (Kasten) bzw. Placebo eingeführt und dann während $4 \mathrm{~h}$ die Sekretion gemessen.

Nach einer weiteren achttägigen Washout-Phase wurde der Versuch im Crossover wiederholt, d.h., dieses Mal war die Gruppe A die Placebound die Gruppe B die Verumgruppe. 
Kasten. Das Prüfpräparat (in der Schweiz unter dem Markennamen Hepa $S^{\circledR}$ erhältlich)

Beim Prüfpräparat handelte es sich um
Kapseln mit $320 \mathrm{mg}$ Artischocken-
blätterextrakt (DEV $4,5-5: 1)$. Die
verwendete Charge enthielt 0,38\%
Caffeoylchinasäurederivate, 0,16\% Di-
caffeoylsäuren und 0,06\% Cynarin. Die
Kapseln wurden in jeweils 50 ml Wasser
gelöst und appliziert. Der Extrakt ist
unter dem Namen LI 220 bekannt.

Die gemessenen Werte wurden statistisch ausgewertet. Die mittlere Steigerung der Cholerese - berechnet bei allen Probanden in den zwei Prüfphasen - war im Vergleich zur gemessenen Basissekretion in der Verumgruppe zu jedem Messzeitpunkt (30, $60,90,120,150 \mathrm{~min}$ ) verglichen mit Placebo signifikant grösser (nach 90 min: $\mathrm{p}<0,01$; nach 120 und $150 \mathrm{~min}$ : $\mathrm{p}<0,05)$. Die höchste Steigerung betrug nach $60 \mathrm{~min}$ 151,5\% des Ausgangswerts. Damit erwies sich der Artischockenextrakt in Bezug auf die biliäre Sekretion als klinisch wirksam.

\section{Funktionelle Dyspepsie}

\section{Holtmann et al.}

Die Wirksamkeit des in der Studie von Kirchhof et al. [2] verwendeten Prüfpräparats wurde in einer weiteren Studie von Holtmann et al. [6] bei funktioneller Dyspepsie untersucht und 2003 publiziert. Der Ansatz der Studie war, für funktionelle Dyspepsien eine wirksame Therapie zu finden, die nicht nur bescheidene Vorteile gegenüber Placebo zeigen, wie das bei $\mathrm{H}_{2}$-Rezeptor-Antagonisten oder Cisaprid der Fall ist [7, 8].

\section{Methode}

Die Studie wurde als prospektive, doppelblinde, randomisierte und placebokontrollierte Multicenterstudie angelegt. Während 6 Wochen wurde den Probanden entweder dreimal täglich Artischockenblätterextrakt LI 220 (enthält 320 mg standardisierten Artischockenextrakt) oder Placebo verabreicht.
Primäre Zielvariable: Beurteilung der Patienten in Bezug auf Veränderungen der dyspeptischen Beschwerden, die mit einem Summenscore ( 0 = keine Verbesserung oder Verschlechterung; 1 = leichte Verbesserung; 2 = deutliche Verbesserung; 3 = vollständige Verbesserung) ausgedrückt wurden.

Sekundäre Zielvariablen: Veränderung der individuell von den Patienten festgestellten dyspeptischen Beschwerden, die von den Patienten beurteilte Intensität jedes dyspeptischen Symptoms, deren Veränderung gegenüber Baseline, die Verbesserung der Lebensqualität sowie eine Gesamtbeurteilung der Wirksamkeit des Prüfpräparats durch Probanden und Prüfärzte.

\section{Probanden}

- Einschlusskriterien: Als Probanden wurden 247 Patienten mit einer diagnostizierten funktionellen Dyspepsie rekrutiert, die sich als chronische oder rezidivierende Beschwerden im Oberbauchbereich zeigte, bei denen eine strukturelle Ursache ausgeschlossen werden konnte. $\mathrm{Zu}$ diesen Einschlusskriterien gehörten Oberbauchschmerzen oder Beschwerden, die sich als unangenehmes, aber nicht wirklich schmerzhaftes Gefühl äusserten. Dazu gehörten schnelles Sättigungs- und postprandiales Völlegefühl sowie Blähungen und Übelkeit, die schon während mindestens 2 Monaten bestanden, ohne dass dafür eine strukturelle oder laborgemässe Erklärung gefunden werden konnte.

- Ausschlusskriterien: Nicht in die Studie eingeschlossen wurden $\mathrm{Pa}$ tienten, die gleichzeitig Präparate einnahmen, die Beschwerden des Gastrointestinaltrakts beeinflussten oder eine Wirkung auf diesen ausübten (z.B. $\mathrm{H}_{2}$-Rezeptor-Antagonisten, Protonenpumpenhemmer, Cholagoga, NSAID), und ebenso Patienten mit Symptomen, die in erster Linie einem Reizdarmsyndrom zugeordnet wurden.
Visiten: Die Patienten wurden bei Baseline sowie nach 2, 4 und 6 Behandlungswochen untersucht. Während der Studie wurden durch die Probanden und die Prüfärzte auch unerwünschte Arzneimittelwirkungen (UAW) sowie die Gesamtverträglichkeit des Prüfpräparats beurteilt.

\section{Resultate}

Gesamtveränderung: Die Gesamtverbesserung der dyspeptischen Beschwerden war in der Artischockengruppe zu jedem Zeitpunkt der Studie grösser als in der Placebogruppe. In der ITT(intention to treat)-Auswertung betrug dies 1,6 Einheiten auf dem Summenscore $(\mathrm{p}=0,007)$ sowie in der PPS-Auswertung 1,7 Einheiten $(\mathrm{p}=0,006)$ und war damit signifikant. Die Zahl der Patienten, bei denen während der Studie die Symptome völlig verschwanden, nahm während der Studie zu und war in der Artischockengruppe grösser als in der Placebogruppe.

Subgruppen: Bei den Subgruppen zeigten sich deutliche Unterschiede. Eine Auswertung betraf Patienten, die gleichzeitig an einem Reizdarmsyndrom litten, und solche ohne Symptome eines Reizdarms. Bei Patienten ohne Reizdarmsyndrom wurde eine grössere Abnahme des Summenscores beobachtet $(9,0 \pm 4,6)$ als bei Patienten mit gleichzeitigem Reizdarmsyndrom $(6,32 \pm 4,9)$. Dieser Unterschied war hochsignifikant ( $p<0,001)$. Ein ähnliches Bild bot sich beim gänzlichen Verschwinden der Symptome im Verlauf der Behandlung. Nur 8,9\% der Probanden mit zusätzlichem Reizdarmsyndrom berichteten von einem vollständigen Verschwinden der Symptome, was noch weniger war als in der Placebogruppe, in der 17,9\% der Probanden dies beobachteten. In der Artischockengruppe war dies aber bei 23,3\% aller Probanden der Fall.

Individuelle Symptome: Die Intensitätsverbesserung der individuellen dyspeptischen Symptome gegenüber Baseline sind in Tabelle 1 aufgeführt. Alle sechs erhobenen Symptome verbesserten sich in beiden Gruppen. Bei 
allen Symptomen war die Verbesserung in der Artischockengruppe ausgeprägter als in der Placebogruppe. Bei den Symptomen Völlegefühl, Flatulenz und schnelles Sättigungsgefühl war dieser Unterschied signifikant $(\mathrm{p}<0,05)$. Positivwerte zeigen eine Verbesserung an, Negativwerte eine Verschlechterung.

Lebensqualität: Ein ähnliches Bild zeigte sich in der Beurteilung der Verbesserung der Lebensqualität gemäss Nepean-Dyspepsia-Index (NDI). In beiden Behandlungsgruppen wurde eine Verbesserung festgestellt. NDISummendifferenz aller Symptome gegenüber Baseline: 89,6 \pm 95,8 (Artischocke) und 67,5 \pm 77,3 (Placebo) $(\mathrm{p}=0,05)$. NDI-Beurteilung der Lebensqualität: $-41,1 \pm 47,6$ (Artischocke) und $-24,8 \pm 35,6$ (Placebo) $(\mathrm{p}=0,01)$.

Gesamtwirksamkeit: Die Gesamtwirksamkeit der Behandlung wurde folgendermassen beurteilt: i) Wirksamkeit in der Artischockengruppe: befriedigend, gut oder sehr gut (Patienten 85,5\%/Prüfärzte 84,7\%); ii) Wirksamkeit in der Placebogruppe: befriedigend oder besser (Patienten $68,7 \% /$ Prüfärzte $69,6 \%$ ). Bei beiden Beurteilungsgruppen zeigte sich für das Artischockenpräparat eine signifikant bessere Beurteilung als für Placebo (beide Gruppen $\mathrm{p}<0,02$ ).

$U A W$ : Während der Behandlung wurden 55 UAW in der Artischockengruppe und 25 UAW in der Placebogruppe dokumentiert, von denen $25,7 \%$ gastrointestinale Symptome darstellten. Die meisten von ihnen waren mild oder mässig und verschwanden von selbst wieder. 4 Probanden (je 2 aus beiden Gruppen) brachen die Studie wegen dieser UAW ab.

\section{Meier und Brignoli}

Eine weitere Studie über die Wirksamkeit des Artischockenblätterpräparats LI 220 bei funktioneller Dyspepsie wurde 2005 in dieser Zeitschrift [9] veröffentlicht und soll deshalb nur noch kurz erwähnt werden:

Die Studie wurde als offene, prospektive und multizentrische Phase-
Tab. 1. Veränderung der Intensität der dyspeptischen Symptome gegenüber Baseline

\begin{tabular}{llll}
\hline Symptom & Artischocke & Placebo & $\mathrm{p}$ \\
\hline Völlegefühl & $6,6 \pm 5,6$ & $4,5 \pm 5,8$ & 0,0050 \\
Flatulenz & $6,6 \pm 5,8$ & $4,7 \pm 5,5$ & 0,0112 \\
Schnelles Sättigungsgefühl & $5,3 \pm 5,9$ & $3,1 \pm 5,8$ & 0,0032 \\
Nausea & $4,3 \pm 5,1$ & $3,9 \pm 5,2$ & 0,5029 \\
Erbrechen & $1,0 \pm 2,6$ & $1,0 \pm 3,0$ & 0,8369 \\
Epigastrische Beschwerden & $7,7 \pm 5,5$ & $6,7 \pm 5,7$ & 0,1786 \\
\hline
\end{tabular}

IV-Studie angelegt, diente also zur weiteren Überprüfung der Wirksamkeit und Sicherheit des Studienpräparats nach dessen Markteinführung. In die Studie wurden 169 Patienten aufgenommen, die seit mindestens $3 \mathrm{Mo}-$ naten an rezidivierender funktioneller Dyspepsie litten. Sie erhielten während 6 Wochen $3 \times$ täglich 2 Kapseln des Prüfpräparats. In die ITT-Auswertung konnten 144 Probanden aufgenommen werden. Bei 74\% dieser Probanden verbesserten sich die Häufigkeit und der Schweregrad der einzelnen Symptome. Besonders zeigte sich dies bei den spezifischen Symptomen wie Völlegefühl, Blähungen, Nausea, rasches Sättigungsgefühl und Schmerzen in der Magengegend. Auch die Alltagsaktivitäten waren nach der 6-wöchigen Behandlung weniger stark gestört als vorher. Zwischen den verschiedenen Subgruppen konnte kein Wirksamkeitsunterschied festgestellt werden. Aus diesen Resultaten schlossen die Autoren, dass sich der Artischockenblätterextrakt LI 220 zur Behandlung der funktionellen Dyspepsie, unabhängig vom Dyspepsie-Typ, eignet und eine geeignete Alternative zu den üblich eingesetzten Arzneimitteln darstellt.

\section{Fazit}

Die hier vorgestellten pharmakologischen und klinischen Studien mit dem standardisierten Artischockenblätterextrakt LI 220 zeigen, dass dieser die Cholerese steigert und bei funktioneller Dyspepsie die Gesamtheit der Symptome signifikant verbessert. LI 220 ist gegenüber Placebo bei der Verminderung der Gesamtheit der dyspeptischen Symptome überlegen. In Bezug auf die individuellen dyspeptischen Symptome zeigten so- wohl die Studie von Holtmann et al. [6] als auch diejenige von Meier und Brignoli [9] eine signifikante Verbesserung bei Flatulenz, Völlegefühl und raschem Sättigungsgefühl. Meier und Brignoli beobachteten eine solche Signifikanz auch bei Nausea und Schmerzen in der Bauchgegend. Beide Studien dokumentierten zudem eine deutliche Abnahme der dyspeptisch bedingten Beeinträchtigung der täglichen Aktivitäten sowie eine gute Verträglichkeit des Prüfpräparats.

Diese Studien zeigen daher, dass das Artischockenblätterpräparat LI 220 gut verträglich und zur Behandlung dyspeptischer Beschwerden sehr geeignet ist und daher eine valable natürliche Alternative zu den üblicherweise verwendeten Arzneimitteln darstellt.

\section{Literatur}

1 Bolli R: Die Artischocke. Phytotherapie 2004; 4:14-18.

2 Kirchhof $\mathrm{R}$, et al: Increase in choleresis by means of artichoke extract. Phytomedicine 1994;1:107-115.

3 Kupke D, et al: Prüfung der choleretischen Aktivität eines pflanzlichen Cholagogums. Z Allg Med 1991;67:1046-1058.

4 Qiang Z, et al: Artichoke extract lowered plasma cholesterol and increased fecal bile acids in Golden Syrian hamsters. Phytother Res 2012; 26:1048-1052.

5 Gebhard R: Inhibition of cholesterol biosynthesis in HepG2 cells by artichoke extracts is reinforced by glycosidase pretreatment. Phytother Res 2002;16:368-372.

6 Holtmann G, et al: Efficacy of artichoke leaf extract in the treatment of patients with functional dyspesia: a six-week placebo-controlled, double-blind, multicentre trial. Aliment Pharmacol Ther 2003;18:1099-1105.

7 Talley NJ, et al: Randomized, double-blind, placebo-controlled crossover trial of cimetidine and pirenzepine in nonulcer dyspepsia. Gastroenterology 1986;91:149-156.

8 Talley NJ, et al: Efficacy of omeprazole in functional dyspepsia: double-blind randomised placebo-controlled trials (the Bond and Opera studies). Aliment Pharmacol Ther 1998;12: 1055-1065.

-9 Meier R, Brignoli R: Artischockenblätterextrakt bei Funktioneller Dyspepsie: Ergebnisse einer offenen, prospektiven, multizentrischen Phase-IV-Studie. Schweiz Zschr Ganzheitsmedizin 2005;17:216-221. 